\title{
A reconciliação de dados na resolução de problemas industriais
}

\author{
Data reconciliation in solving industrial problems \\ R. L. S. França ${ }^{1 *}$; D. F. S. Souza ${ }^{2}$; A. M. Oliveira Jr. ${ }^{1}$ \\ ${ }^{1}$ Programa de Pós-Graduação em Engenharia Química, Universidade Federal de Sergipe, 49100-000, São \\ Cristóvão- Sergipe, Brasil \\ ${ }^{2}$ Departamento de Engenharia Química, Universidade Federal do Rio Grande do Norte, 59078-970, Natal- Rio \\ Grande do Norte, Brasil \\ *reginaquimica@gmail.com
}

(Recebido em 31 de janeiro de 2016; aceito em 12 de abril de 2016)

\begin{abstract}
Variáveis industriais possuem importância fundamental para êxito em processos, porém muitas das vezes se estas não forem medidas corretamente podem carregar consigo erros que tendem a modificar suas características e até prejudicar o resultado final. O presente estudo consistiu na aplicação da técnica da reconciliação de dados $(\mathrm{RD})$, uma ferramenta matemática para o ajuste de medidas que tendem a obedecer às restrições mássicas e energéticas dos processos, em dois modelos adaptados de plantas industriais operando em sistema estacionário com restrições lineares. No primeiro caso, uma indústria de produtos intermediários de fertilizantes onde foi aplicada como etapa principal a classificação das variáveis para observabilidade do sistema. No segundo caso, uma planta de polimerização de um pólo petroquímico onde foram observadas as variáveis utilizando duas funções robustas que pretendiam atenuar erros em medidas. Os resultados obtidos mostraram que a técnica concebida com base em modelo estatístico resultou na convergência do método com um menor número de iterações, correção dos dados medidos e consequentemente redução da variância das variáveis importantes para o processo. O uso dessa técnica para resolução de problemas da engenharia química torna-se valorosa tendo em vista a grande quantidade de instrumentos encontrados nas plantas industriais, que geram milhares de medidas que necessitam de um elevado nível de confiabilidade para uma operação adequada da planta.

Palavras-chave: Reconciliação de dados, variáveis, processos industriais.
\end{abstract}

Industrial variables have fundamental importance for successful processes; though often if they are not measured correctly can carry with errors that tend to modify their characteristics and to prejudice the results. This study was the technique of application data reconciliation (DR) a mathematical tool for adjustment measures that tend to conform to the mass and energy constraints of processes in two adapted models of industrial plants operating in steady state system with linear constraints. In the first case an intermediate fertilizer products industry where it is applied as a main step of the classification variables for observability of the system. In the second polymerization, plant of petrochemical complex variables observed using two robust functions intended to mitigate errors in measurements. The results showed that the art designed based on the statistical model resulted in the convergence of the method with a smaller number of iterations, correction of measured data and hence reduce the variance of the important variables for the process. The use of this technique for the chemical engineering problem solving becomes valuable in view of the large number of instruments found in industrial plants, which generate thousands of measures that require a high level of reliability for proper operation of the plant.

Keywords: Data Reconciliation, variables, industrial processes.

\section{INTRODUÇÃO}

Todas as variáveis de processos industriais necessitam diariamente ser observadas objetivando o correto funcionamento dos sensores de medição, a incidência de erros e a otimização do sistema gerencial. Esses dados de processos gerados por instrumentos podem possuir erros de medição e/ou erros grosseiros, que se devem a problemas em equipamentos. Em virtude disso, alguns conjuntos de dados não obedecem às leis de conservação de massa e balanços de energia [1]. 
Algumas técnicas são utilizadas com o intuito de analisar variações em sistemas como a estimação de parâmetros, modelagem e simulação de processos em ambientes computacionais e a reconciliação de dados (RD) que dentre uma gama de aplicações, permite o ajuste ótimo de medidas e estimativas com base em redundância espacial e no modelo, geralmente balanços de massa e/ou energia [2].

Em plantas industriais, alguns problemas causados pela má qualidade dos dados medidos devem ser identificados. Neste estudo, problemas como vazamentos de vapor, drenagens de condensados, perda de eficiência e riscos operacionais oneravam determinados processos causando grandes danos.

\subsection{Reconciliação de dados}

A reconciliação de dados foi inicialmente proposta para calcular o balanço de massa em processos operando em estado estacionário [2-8]. Vaclávek (1969) [9], em um estudo posterior, aprimorou os dados medidos através da derivação de um algoritmo que classificou as correntes medidas e as correntes não medidas de acordo com as propriedades topológicas no esquema de balanço. As medidas foram dispostas em dois grupos, o primeiro com medidas que podiam ter seu valor obtido através de outras correntes medidas sem necessidade de medição direta e o outro grupo que somente teria valor através de medidas diretas. Romagnoli e Stephanopoulos (1981) [10] apresentaram um método para identificar a fonte de erros através da eliminação seriada de uma ou mais medidas de cada vez e utilizaram o método dos mínimos quadrados para a obtenção dos ajustes, tendo como restrições os balanços de massa nodais.

\subsection{Classificação de variáveis}

Os precursores do estudo sobre classificação de variáveis foram Vaclavék e Loucka (1976) [11] que as caracterizaram em redundantes e observáveis. Para Bagajewicz (2001) [12], a medição de uma variável é não redundante se após a remoção desta medição a variável não é observável, isto é, não pode ser calculada usando uma equação de equilíbrio envolvendo as outras medições do sistema.

Uma variável observável é aquela não medida que pode ser calculada através de variáveis medidas ligadas ao modelo de processo. Uma variável não observável é aquela que não está disponível qualquer informação no processo. Stanley e Mah (1981) [13] apresentaram dois algoritmos para determinação das observabilidades local e global, redundância de variáveis individuais e medições de processos baseadas em técnicas de análise teórico-gráfica. Stanley e Mah (1981) [14] desenvolveram uma teoria sobre observabilidade e redundância aplicável a sistemas no estado estacionário, através de analogias com sistemas dinâmicos. Para restrições e medições lineares, as condições para observabilidade local também valeram para a observabilidade global e o sistema foi subdividido em subsistemas (redundante, estritamente observável e não observável). Oliveira Jr. et al. (2011) [15] propuseram uma algoritmo de classificação de um processo de polimerização visando o conhecimento da planta para solução de pequenas oscilações do sistema.

\subsection{Funções robustas na $\mathrm{RD}$}

Funções robustas são aquelas com capacidade de atenuar a influência dos erros grosseiros em dados medidos com base em modelos estatísticos. Arora e Biegler (2001) [16] formularam no problema da reconciliação de dados clássica a solução dos erros tanto grosseiros quanto sistemáticos através do uso de estimadores robustos como o Hampel de três partes, o Mestimador e a função Fair, aliados ao método de "Branch and Bound" para resolução com estratégia automatizada. O estimador de três partes de Hampel foi o que apresentou maior robustez e com um ponto de corte que contribuiu com a reconciliação simultaneamente sem a necessidade do uso de análise exploratória. Ozyurt e Pike (2004) [17] aplicaram sete funções robustas para a RD visando detectar erros grosseiros através de métodos estatísticos baseados em distribuição combinada com auxílio de método de Monte Carlo na obtenção das constantes e 
das eficiências relativas dos estimadores robustos. As funções aplicadas foram Mínimos quadrados ponderados, Normal Contaminada, Cauchy, Logística. Lorenziana, Fair e o Mestimador Hampel redescendente. Outros autores buscaram implementar essas funções robustas no problema de estimação e reconciliação de dados. Zhang et al. (1995) [18] aplicaram a Normal Contaminada em dados reais e estado estacionário, assim como Bourouis et al. (1998) [19], Schladt e Hu (2007) [20], Lid e Skogestad (2008) [21] e Chen et al. (2013) [22] em unidade de dessalinização por separação flash com restrições não lineares. Jin et al. (2012) [23] implementaram um novo estimador robusto chamado de New Target para reconciliação de dados robustos. A função proposta buscou detectar pequenos erros grosseiros nas medidas já que as outras funções estudadas não possuíam essa característica. Os autores concluíram que a reconciliação de dados utilizando a New Target obteve dados mais precisos principalmente quando existia um ou dois erros grosseiros nas medidas, o que representou maior robustez entre as funções.

Neste trabalho foram utilizados dois exemplos adaptados de plantas industriais sendo que no primeiro caso observou-se apenas a importância da classificação de variáveis para simplificação de um complexo sistema industrial, e no segundo a reconciliação de dados com o uso de funções robustas cujo objetivo foi analisar a capacidade de atenuar os erros grosseiros presentes nas medidas reais.

\section{METODOLOGIA}

A metodologia aplicada nesse artigo baseia-se em dois estudos de casos reais que por critério de sigilo industrial informações a respeito das empresas não serão divulgadas.

\subsection{Estudo de caso}

\section{CASO 1. Classificação de variáveis em unidade industrial}

O primeiro caso trata de um processo em uma fábrica de fertilizantes nitrogenados apresentando um sistema modelo da classificação de variáveis em unidade industrial. É fundamental o conhecimento do processo a ser aplicado a reconciliação dos dados pois a classificação de variáveis deverá verificar se todas as variáveis são medidas e, não sendo, quais poderão ser observadas por serem importante para o sistema e quais precisarão de atenção (não observáveis). A etapa de classificação de variáveis deve seguir os passos abaixo conforme Oliveira Jr. et al. (2011) [15]:

1) Exibir um fluxograma que deve possuir "nós" que são divisores, misturadores e operações, bem como sensores de medição das variáveis;

2) Construir uma tabela com as variáveis porque através dela serão concebidas as equações do modelo para o sistema.

3) Propor um modelo matemático através de uma matriz de ocorrência que pode ser composto de equações algébricas ou algébrico-diferenciais. Neste estudo, o modelo concebido baseou-se em equações algébricas em que as linhas corresponderam às equações e as colunas todas as variáveis do processo. Os elementos da matriz de ocorrência correspondem quando $\mathbf{M}$ preenchida em $\mathrm{M}_{\mathrm{ij}}=1$ se a variável $\mathrm{j}$ ocorrer na equação e $\mathrm{M}_{\mathrm{ij}}=0$ caso ocorresse o contrário.

4) De posse da matriz de ocorrência é gerado um algoritmo de classificação para análise de todas as variáveis pertencentes ao modelo.

- A coluna referente à variável medida $\mathrm{x}_{\mathrm{n}}$ é zerada, de forma que Min $=0$ para todo $\mathrm{i}$. Repete-se para cada uma das variáveis medidas.

- Identificam-se as linhas de zero da matriz M. As linhas indicam equações redundantes que contém apenas variáveis medidas.

- Aplicam-se as técnicas de comparação para identificação dos conjuntos de $\mathbf{n}$ equações e $\mathbf{n}$ incógnitas ( $\mathbf{n}$ linhas de $\mathbf{M}$ que apresentem as mesmas $\mathbf{n}$ ocorrências), partindo de $\mathbf{n}=\mathbf{2}$. Se realizada corretamente, as $\mathbf{n}$ variáveis identificadas são ditas observáveis, caso contrário, as variáveis remanescentes em $\mathbf{M}$ são ditas não observáveis. 
- Os dados da entrada desse algoritmo com base nas variáveis do sistema devem conter a quantidade; a identificação; o número total delas; o número de equações levantadas; e a matriz de ocorrência.

Na Figura 1 é apresentado o fluxograma adaptado do processo analisado.

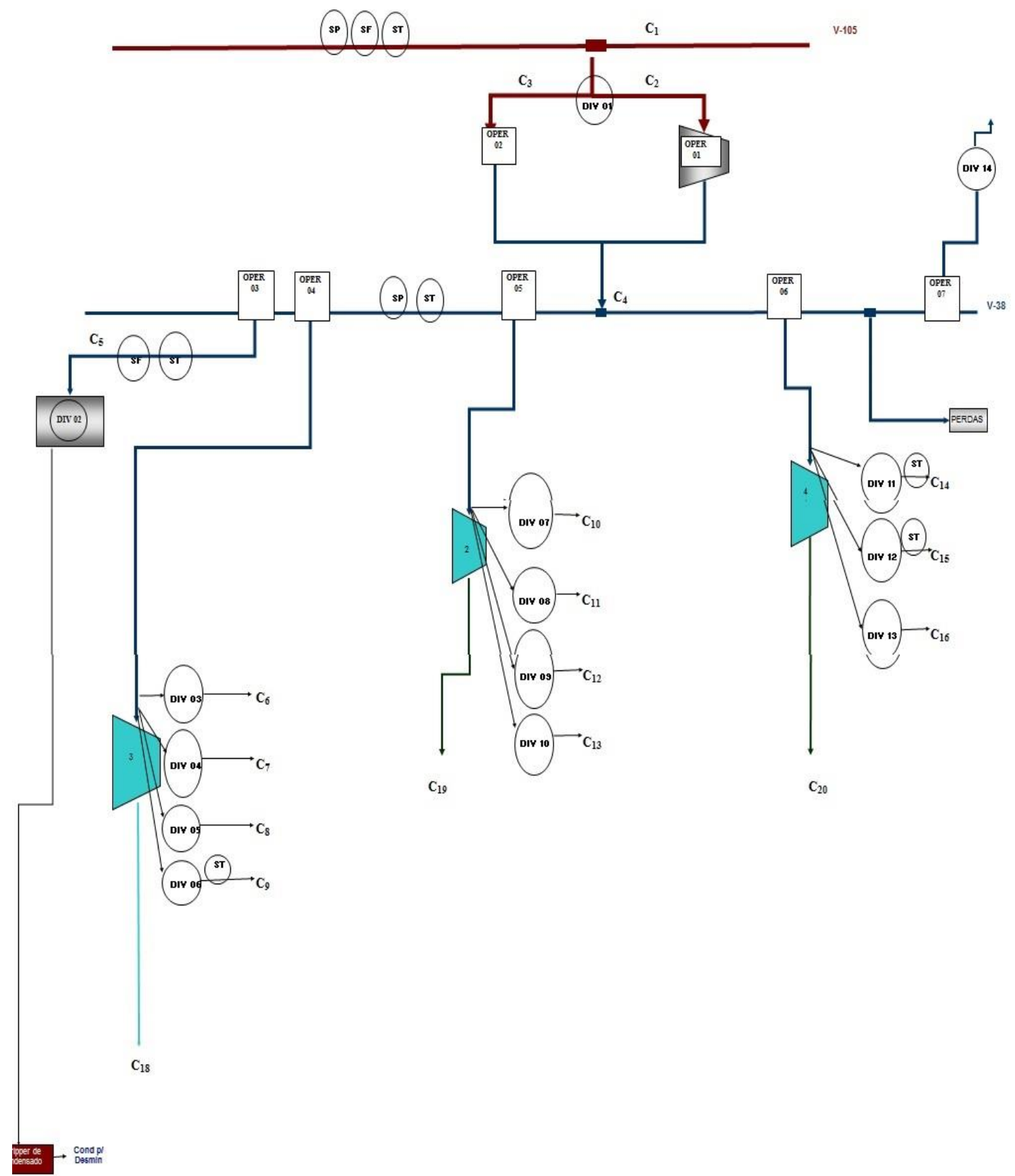

Figura 1. Fluxograma adaptado de processo industrial de geração de vapor.

Os "nós" do fluxograma relatam os divisores (DIV), operadores (OPER), sensores de fluxo (SF), de pressão (SP) e temperatura (ST). As operações de geração para consumo de vapor e acionamento de turbo máquinas na unidade são representadas pelas Operações 01, 03, 04, 05, e 06 e pelas correntes c4 a c21. A Operação 02 é uma corrente alternativa e somente é acionada caso a Operação 01 esteja impossibilitada de funcionar. A corrente c1 é constituída da geração de vapor a uma dada pressão e as correntes c2 e c3 que contêm a geração desse vapor a uma pressão menor, possuem medidores de vazão, temperatura e pressão. A Operação 03 contém o 
stripper do condensado para desmineralização onde é gerado hidrogênio identificado como c17. A Operação 07 recolhe uma parte do vapor para usos internos e a maior quantidade para fora da unidade através do sistema de vácuo, corrente c21.

\section{CASO 2. Aplicação de funções robustas em planta de polimerização}

O segundo caso retrata dados reais de um processo de polipropileno (Figura 2) com características estacionárias, será apresentada uma simplificação do processo para avaliação das funções robustas. O proposto foi a apresentação da comparação das funções robustas em um modelo estacionário de um processo industrial através do estudo do balanço mássico em relação ao reator de polimerização.



Figura 2. Processo de polimerização simplificado

A polimerização do polipropileno em massa é um processo em lama no qual o diluente é o próprio monômero (contaminado por propano) que é alimentado na fase líquida. O doador de elétrons (PEEB) e outros agentes são introduzidos mediante uma corrente de alimentação também em fase líquida. São utilizados reatores CSTR em combinação com catalisadores Ziegler-Natta de quarta geração $\left(\mathrm{TiCl}_{4} / \mathrm{MgCl}_{2}+\mathrm{PEEB}+\mathrm{TEA}\right)$ e o TEA é co-catalisador. A corrente de saída do reator é composta basicamente de propano, propeno e polipropileno [24].

Nesse processo foram classificadas as variáveis medidas e as variáveis não medidas foram estimadas com as funções robustas, são elas:

- Variáveis medidas: Vazão de alimentação de propeno fresco $(\boldsymbol{m e})$, vazão de alimentação de catalisador $(\boldsymbol{m} \boldsymbol{C A T})$, vazão de alimentação de trietil alumínio ( $\boldsymbol{m T E A})$, vazão de alimentação de p-etiletóxido-benzoato $(\boldsymbol{m P E E B})$ e vazão de polipropileno produzido $(\boldsymbol{m P o l})$.

- Variáveis não medidas: Vazão de purga (mpurga), massa de propeno no reator $(\boldsymbol{P e})$, massa de propano no reator $(\boldsymbol{P a})$, massa de polímero no reator $(\boldsymbol{P o l})$, massa de catalisador $(\boldsymbol{C A T})$, massa de TEA (TEA) e massa de PEEB (PEEB).

As equações (1) a (7) apresentam o modelo matemático baseado no balanço mássico do processo analisado, as variâncias medidas estão apresentadas na Tabela 1 e os parâmetros fixos na Tabela 2. Os valores dos parâmetros das Tabelas 1 e 2 foram apresentados por Prata et al. (2010) [25].

\section{Balanço de massa para o Propano:}

$$
m_{e} \cdot w_{a}-\left(\frac{P a}{P a+P e}\right) \cdot m_{\text {purga }}=0
$$


Balanço de massa para o Propeno:

$m_{e} \cdot\left(1-w_{a}\right)-R_{p o l}-\left(\frac{P e}{P a+P e}\right) \cdot \mathrm{m}_{\text {purga }}=0$

Balanço de massa para o Polipropileno:

$R_{p o l}-m_{\text {pol }}=0$

Cálculo da taxa de polimerização (produção de polipropileno):

$R_{p o l}=K p \cdot\left(\frac{C A T \cdot P e}{M}\right)$

Balanço de massa para o Catalisador:

$m_{C A T}-\left(\frac{C A T}{P O L}\right) \cdot m_{p o l}-K d . C A T=0$

Balanço de massa para o PEEB:

$m_{P E E B}-\left(\frac{P E E B}{P o l}\right) \cdot m_{p o l}=0$

Balanço de massa para o TEA:

$m_{T E A}-\alpha \cdot\left(\frac{T E A}{P e+P a}\right) \cdot \mathrm{m}_{\mathrm{purga}}-(1-\alpha) \cdot\left(\frac{T E A}{P o l}\right) \cdot m_{p o l}=0$

Tabela 1. Variâncias para as variáveis medidas do processo de polimerização.

\begin{tabular}{ccc}
\hline Variáveis & Descrição & Variância( $\mathbf{k g} / \mathbf{h})$ \\
\hline$m_{e}$ & Vazão de propeno & $3,600 \cdot 10^{5}$ \\
$m_{C A T}$ & Vazão do catalisador & $2,500 \cdot 10^{-3}$ \\
$m_{T E A}$ & Vazão de TEA & $2,500 \cdot 10^{-3}$ \\
$m_{P E E B}$ & Vazão de PEEB & $2,500 \cdot 10^{-3}$ \\
$m_{\text {Pol }}$ & Vazão de polímero & $3,600 \cdot 10^{5}$ \\
\hline
\end{tabular}

Tabela 2. Valores dos parâmetros fixos do modelo

\begin{tabular}{clc}
\hline Parâmetros & \multicolumn{1}{c}{ Descrição } & Valores \\
\hline$\alpha$ & Fator de reciclo de TEA & 0,0002 \\
$K d$ & Constante de iniciação & 2 \\
$K p$ & Constante cinética de propagação & 5,5 \\
$w_{a}$ & Fração mássica de propano & $0,004 \mathrm{~kg} / \mathrm{kg}$ \\
\hline
\end{tabular}

Através desse modelo foi realizada a reconciliação de dados das variáveis medidas e estimação das variáveis não medidas que são observáveis com a aplicação das funções robustas escolhidas.

Reconciliar dados utilizando funções robustas é apresentar uma modificação da função de mínimos quadrados ponderados para implementação de um estimador com função objetivo de verossimilhança [26], conforme a Equação (8):

$\min \sum_{i} \rho\left(\frac{y_{i}-x_{i}}{\sigma_{i}}\right)$ 
em que $\rho$ é a função robusta, $y_{i}$ são os valores medidos, $x_{i}$ os valores reconciliados e $\sigma_{i} 0$ desvio padrão e o erro padrão é expresso pela Equação (9):

$$
\varepsilon_{i}=\frac{\left(y_{i}-x_{i}\right)}{\sigma_{i}}
$$

Duas funções robustas foram aplicadas nesse estudo de caso: Normal Contaminada Equação (10) e New Target Equação (11).

\section{Normal contaminada}

$$
-\ln \left\{\left(1-p_{C N}\right) \exp \left(-\frac{\varepsilon_{i}^{2}}{2}\right)+\frac{p_{C N}}{b_{C N}} \exp \left(-\frac{\varepsilon_{i}^{2}}{2 b_{C N}^{2}}\right)\right\}
$$

\section{New Target}

$$
\begin{cases}\frac{c^{2}}{6}\left[1-\left[1-a \frac{r^{2}}{c^{2}}\right]^{3}\right] \text { se }|r| \leq c \\ A \ln \frac{r^{2}}{c^{2}}+B & \text { se }|r| \geq c\end{cases}
$$

O valor das constantes foram extraídas de Ozyurt e Pike (2004) [17] e Jin et al. (2012) [23] (Tabela 3).

Tabela 3. Valor das constantes para diferentes funções $\rho$ com valores de eficiência de $95 \%$

\begin{tabular}{ll}
\hline \multicolumn{1}{c}{ Função $\rho$} & \multicolumn{1}{c}{ Valor das constantes } \\
\hline Normal Contaminada & $\mathrm{b}_{\mathrm{CN}}=10, \mathrm{p}_{\mathrm{CN}}=0.235$ \\
New Target & $\mathrm{c}=3, \mathrm{a}=0.65$ \\
\hline
\end{tabular}

Funções como essas possuem constantes como uma função de eficiência assintótica. Entretanto, essas variações assintóticas "apresentam apenas indicações brutas para as verdadeiras variâncias" no tamanho da amostra finita [27]. Portanto, a aproximação finita no tamanho das amostras e consequentemente a sua relativa eficiência é obtida através de simulações de Monte Carlo com corridas variando de 28 a 2.000 simulações [17]. A escolha dessas duas funções deve-se a sua forma funcional que consegue atenuar o valor da função em relação a grandes variações de erro.

A análise das funções robustas foi realizada em ambiente computacional com o uso de função que visava encontrar o mínimo de uma função multivariável sujeito a restrições não lineares de igualdades e desigualdades. Essa função após formulação da estrutura do modelo e resolução de otimização retorna os valores reconciliados.

\section{RESULTADOS E DISCUSSÃO}

Para uma rede de processos operando no estado estacionário, as variáveis são restringidas pelas equações de conservação. Para um dado processo com conjunto de instrumentos distribuídos ao longo da planta, caso se realize uma mudança de variável sem ser detectada (observada) pelos instrumentos, a variável é dita não observável. Usando esta definição, toda variável seria observável se todas as variáveis fossem medidas. Como isso quase nunca acontece por razões de custo, conveniência ou viabilidade técnica, o que se tem na realidade é um conjunto de medições incompletas que gostaríamos de conhecer se cada variável é ou não observável. Esse problema é chamado de classificação de observabilidade [15]. 


\section{Caso 1}

Devido ao conjunto incompleto de instrumentos em uma unidade industrial, as variáveis não medidas são divididas em determináveis ou estimáveis e indetermináveis ou inestimáveis. As variáveis não medidas são determináveis ou estimáveis, se os valores podem ser calculados usando as medidas existentes. Já as variáveis medidas são classificadas em redundantes e não redundantes. Uma medida é redundante se a mesma permanece determinável quando a sua medida é excluída [15].

Com as informações pertinentes ao processo, é concebida a Tabela 4 onde são apresentadas as variáveis de interesse através da identificação do processo.

Tabela 4 - Identificação de Correntes e de Variáveis - Projeto

\begin{tabular}{|c|c|c|c|}
\hline Corrente & Variável de Interesse & Var número & Característica \\
\hline \multirow[t]{3}{*}{$\mathrm{C} 1$} & Vazão Mássica & $\mathrm{x} 1$ & Medida \\
\hline & Pressão & $\mathrm{x} 2$ & Medida \\
\hline & Temperatura & $\mathrm{x} 3$ & Medida \\
\hline \multirow[t]{3}{*}{$\mathrm{C} 2$} & Vazão Mássica & $\mathrm{x} 4$ & Não Medida \\
\hline & Pressão & $\mathrm{x} 5$ & Medida \\
\hline & Temperatura & $x 6$ & Medida \\
\hline $\mathrm{C} 3$ & Vazão Mássica & $\mathrm{x} 7$ & Não Medida \\
\hline \multirow[t]{3}{*}{$\mathrm{C} 4$} & Vazão Mássica & $\mathrm{x} 8$ & Não Medida \\
\hline & Temperatura & $\mathrm{x} 9$ & Medida \\
\hline & Pressão & $\mathrm{x} 10$ & Medida \\
\hline \multirow[t]{3}{*}{ C5 } & Vazão Mássica & $\mathrm{x} 11$ & Não Medida \\
\hline & Pressão & $\mathrm{x} 12$ & Medida \\
\hline & Temperatura & $\mathrm{x} 13$ & Medida \\
\hline C6 & Vazão Mássica & $\mathrm{x} 14$ & Não Medida \\
\hline $\mathrm{C} 7$ & Vazão Mássica & $\mathrm{x} 15$ & Não Medida \\
\hline $\mathrm{C} 8$ & Vazão Mássica & $\mathrm{x} 16$ & Não Medida \\
\hline \multirow[t]{2}{*}{ C9 } & Vazão Mássica & $\mathrm{x} 17$ & Não Medida \\
\hline & Temperatura & $\mathrm{x} 18$ & Medida \\
\hline $\mathrm{C} 10$ & Vazão Mássica & $\mathrm{x} 19$ & Não Medida \\
\hline $\mathrm{C} 11$ & Vazão Mássica & $\mathrm{x} 20$ & Não Medida \\
\hline $\mathrm{C} 12$ & Vazão Mássica & $\mathrm{x} 21$ & Não Medida \\
\hline $\mathrm{C} 13$ & Vazão Mássica & $\mathrm{x} 22$ & Não Medida \\
\hline \multirow[t]{2}{*}{$\mathrm{C} 14$} & Vazão Mássica & $\mathrm{x} 23$ & Não Medida \\
\hline & Temperatura & $\mathrm{x} 24$ & Medida \\
\hline \multirow[t]{2}{*}{$\mathrm{C} 15$} & Vazão Mássica & $\mathrm{x} 25$ & Não Medida \\
\hline & Temperatura & $\mathrm{x} 26$ & Medida \\
\hline $\mathrm{C} 16$ & Vazão Mássica & $\mathrm{x} 27$ & Não Medida \\
\hline $\mathrm{C} 17$ & $\begin{array}{l}\text { Condensado para } \\
\text { desmineralização }\end{array}$ & $\mathrm{x} 28$ & Não Medida \\
\hline $\mathrm{C} 18$ & Exausto V-4 & $\mathrm{x} 29$ & Medida \\
\hline $\mathrm{C} 19$ & Exausto V-14 & $\mathrm{x} 30$ & Medida \\
\hline $\mathrm{C} 20$ & Exausto V-14 & x31 & Medida \\
\hline $\mathrm{C} 21$ & Descarga superfície & x32 & Não Medida \\
\hline
\end{tabular}

É possível observar que, partindo de um sistema complexo, foram classificadas 32 variáveis e a partir delas foi concebido um modelo com as equações algébricas do balanço mássico para a formação da matriz de ocorrência A de todas as variáveis do processo apresentada na Figura 3. 
Números de variáveis medidas: 15

Variáveis medidas: 1, 2, 3, 5, 6, 9, 10, 12, 13, 18, 24, 26, 29, 30, 31

Número de equações: 15

Número de variáveis: 32

$\begin{array}{lcccccccccccccccccccccccccccccccccccc} & 1 & 2 & 3 & 4 & 5 & 6 & 7 & 8 & 9 & 10 & 11 & 12 & 13 & 14 & 15 & 16 & 17 & 18 & 19 & 20 & 21 & 22 & 23 & 24 & 25 & 26 & 27 & 28 & 29 & 30 & 31 & 32 \\ \text { Eq.12 } & 1 & 0 & 0 & 1 & 0 & 0 & 0 & 0 & 0 & 0 & 0 & 0 & 0 & 0 & 0 & 0 & 0 & 0 & 0 & 0 & 0 & 0 & 0 & 0 & 0 & 0 & 0 & 0 & 0 & 0 & 0 & 0 \\ \text { Eq.13 } & 0 & 0 & 0 & 1 & 0 & 0 & 0 & 1 & 0 & 0 & 0 & 0 & 0 & 0 & 0 & 0 & 0 & 0 & 0 & 0 & 0 & 0 & 0 & 0 & 0 & 0 & 0 & 0 & 0 & 0 & 0 & 0 \\ \text { Eq.14 } & 0 & 0 & 0 & 0 & 0 & 0 & 0 & 1 & 0 & 0 & 1 & 0 & 0 & 0 & 0 & 0 & 0 & 0 & 0 & 0 & 0 & 0 & 0 & 0 & 0 & 0 & 0 & 1 & 0 & 0 & 0 & 0 \\ \text { Eq.15 } & 0 & 0 & 0 & 0 & 0 & 0 & 0 & 1 & 1 & 0 & 1 & 0 & 1 & 0 & 0 & 0 & 0 & 0 & 0 & 0 & 0 & 0 & 0 & 0 & 0 & 0 & 0 & 1 & 0 & 0 & 0 & 0 \\ \text { Eq.16 } & 0 & 0 & 0 & 0 & 0 & 0 & 0 & 1 & 0 & 0 & 0 & 0 & 0 & 1 & 1 & 1 & 1 & 0 & 0 & 0 & 0 & 0 & 0 & 0 & 0 & 0 & 0 & 0 & 1 & 0 & 0 & 0 \\ \text { Eq.17 } & 0 & 0 & 0 & 0 & 0 & 0 & 0 & 1 & 0 & 0 & 0 & 0 & 0 & 1 & 0 & 0 & 0 & 0 & 0 & 0 & 0 & 0 & 0 & 0 & 0 & 0 & 0 & 0 & 0 & 0 & 0 & 0 \\ \text { Eq.18 } & 0 & 0 & 0 & 0 & 0 & 0 & 0 & 1 & 0 & 0 & 0 & 0 & 0 & 0 & 1 & 0 & 0 & 0 & 0 & 0 & 0 & 0 & 0 & 0 & 0 & 0 & 0 & 0 & 0 & 0 & 0 & 0 \\ \text { Eq.19 } & 0 & 0 & 0 & 0 & 0 & 0 & 0 & 1 & 0 & 0 & 0 & 0 & 0 & 0 & 0 & 1 & 0 & 0 & 0 & 0 & 0 & 0 & 0 & 0 & 0 & 0 & 0 & 0 & 0 & 0 & 0 & 0 \\ \text { Eq.20 } & 0 & 0 & 0 & 0 & 0 & 0 & 0 & 1 & 0 & 0 & 0 & 0 & 0 & 0 & 0 & 0 & 1 & 0 & 0 & 0 & 0 & 0 & 0 & 0 & 0 & 0 & 0 & 0 & 0 & 0 & 0 & 0 \\ \text { Eq.21 } & 0 & 0 & 0 & 0 & 0 & 0 & 0 & 1 & 1 & 0 & 0 & 0 & 0 & 0 & 0 & 0 & 1 & 1 & 0 & 0 & 0 & 0 & 0 & 0 & 0 & 0 & 0 & 0 & 0 & 0 & 0 & 0 \\ \text { Eq.22 } & 0 & 0 & 0 & 0 & 0 & 0 & 0 & 1 & 0 & 0 & 0 & 0 & 0 & 0 & 0 & 0 & 0 & 0 & 1 & 0 & 1 & 1 & 0 & 0 & 0 & 0 & 0 & 0 & 0 & 1 & 0 & 0 \\ \text { Eq.23 } & 0 & 0 & 0 & 0 & 0 & 0 & 0 & 1 & 0 & 0 & 0 & 0 & 0 & 0 & 1 & 0 & 0 & 0 & 0 & 0 & 0 & 0 & 0 & 0 & 0 & 0 & 0 & 0 & 0 & 0 & 0 & 0 \\ \text { Eq.24 } & 0 & 0 & 0 & 0 & 0 & 0 & 0 & 1 & 0 & 0 & 0 & 0 & 0 & 0 & 0 & 0 & 0 & 0 & 0 & 0 & 0 & 0 & 1 & 0 & 1 & 0 & 1 & 0 & 0 & 0 & 1 & 0 \\ \text { Eq.25 } & 0 & 0 & 0 & 0 & 0 & 0 & 0 & 1 & 1 & 0 & 0 & 0 & 0 & 0 & 0 & 0 & 0 & 0 & 0 & 0 & 0 & 0 & 1 & 1 & 1 & 1 & 1 & 0 & 0 & 0 & 1 & 0 \\ \text { Eq.26 } & 0 & 0 & 0 & 0 & 0 & 0 & 0 & 1 & 0 & 0 & 0 & 0 & 0 & 0 & 0 & 0 & 0 & 0 & 0 & 0 & 0 & 0 & 0 & 0 & 0 & 0 & 0 & 0 & 0 & 0 & 0 & 1\end{array}$

Figura 3. Matriz de ocorrência das variáveis

Analisando a matriz de ocorrência A é gerado um algoritmo de classificação onde as linhas representam as equações e as colunas as variáveis (Figura 4).

$\begin{array}{lcccccccccccccccccccccccccccccccccccccc} & 1 & 2 & 3 & 4 & 5 & 6 & 7 & 8 & 9 & 10 & 11 & 12 & 13 & 14 & 15 & 16 & 17 & 18 & 19 & 20 & 21 & 22 & 23 & 24 & 25 & 26 & 27 & 28 & 29 & 30 & 31 & 32 \\ \text { Eq.12 } & 0 & 0 & 0 & 0 & 0 & 0 & 0 & 0 & 0 & 0 & 0 & 0 & 0 & 0 & 0 & 0 & 0 & 0 & 0 & 0 & 0 & 0 & 0 & 0 & 0 & 0 & 0 & 0 & 0 & 0 & 0 & 0 \\ \text { Eq.13 } & 0 & 0 & 0 & 0 & 0 & 0 & 0 & 0 & 0 & 0 & 0 & 0 & 0 & 0 & 0 & 0 & 0 & 0 & 0 & 0 & 0 & 0 & 0 & 0 & 0 & 0 & 0 & 0 & 0 & 0 & 0 & 0 \\ \text { Eq.14 } & 0 & 0 & 0 & 0 & 0 & 0 & 0 & 0 & 0 & 0 & 1 & 0 & 0 & 0 & 0 & 0 & 0 & 0 & 0 & 0 & 0 & 0 & 0 & 0 & 0 & 0 & 0 & 1 & 0 & 0 & 0 & 0 \\ \text { Eq.15 } & 0 & 0 & 0 & 0 & 0 & 0 & 0 & 0 & 0 & 0 & 0 & 0 & 0 & 0 & 0 & 0 & 0 & 0 & 0 & 0 & 0 & 0 & 0 & 0 & 0 & 0 & 0 & 1 & 0 & 0 & 0 & 0 \\ \text { Eq.16 } & 0 & 0 & 0 & 0 & 0 & 0 & 0 & 0 & 0 & 0 & 0 & 0 & 0 & 0 & 0 & 0 & 0 & 0 & 0 & 0 & 0 & 0 & 0 & 0 & 0 & 0 & 0 & 0 & 0 & 0 & 0 & 0 \\ \text { Eq.17 } & 0 & 0 & 0 & 0 & 0 & 0 & 0 & 0 & 0 & 0 & 0 & 0 & 0 & 0 & 0 & 0 & 0 & 0 & 0 & 0 & 0 & 0 & 0 & 0 & 0 & 0 & 0 & 0 & 0 & 0 & 0 & 0 \\ \text { Eq.18 } & 0 & 0 & 0 & 0 & 0 & 0 & 0 & 0 & 0 & 0 & 0 & 0 & 0 & 0 & 0 & 0 & 0 & 0 & 0 & 0 & 0 & 0 & 0 & 0 & 0 & 0 & 0 & 0 & 0 & 0 & 0 & 0 \\ \text { Eq.19 } & 0 & 0 & 0 & 0 & 0 & 0 & 0 & 0 & 0 & 0 & 0 & 0 & 0 & 0 & 0 & 0 & 0 & 0 & 0 & 0 & 0 & 0 & 0 & 0 & 0 & 0 & 0 & 0 & 0 & 0 & 0 & 0 \\ \text { Eq.20 } & 0 & 0 & 0 & 0 & 0 & 0 & 0 & 0 & 0 & 0 & 0 & 0 & 0 & 0 & 0 & 0 & 0 & 0 & 0 & 0 & 0 & 0 & 0 & 0 & 0 & 0 & 0 & 0 & 0 & 0 & 0 & 0 \\ \text { Eq.21 } & 0 & 0 & 0 & 0 & 0 & 0 & 0 & 0 & 0 & 0 & 0 & 0 & 0 & 0 & 0 & 0 & 0 & 0 & 0 & 0 & 0 & 0 & 0 & 0 & 0 & 0 & 0 & 0 & 0 & 0 & 0 & 0 \\ \text { Eq.22 } & 0 & 0 & 0 & 0 & 0 & 0 & 0 & 0 & 0 & 0 & 0 & 0 & 0 & 0 & 0 & 0 & 0 & 0 & 1 & 0 & 1 & 1 & 0 & 0 & 0 & 0 & 0 & 0 & 0 & 0 & 0 & 0 \\ \text { Eq.23 } & 0 & 0 & 0 & 0 & 0 & 0 & 0 & 0 & 0 & 0 & 0 & 0 & 0 & 0 & 0 & 0 & 0 & 0 & 0 & 0 & 0 & 0 & 0 & 0 & 0 & 0 & 0 & 0 & 0 & 0 & 0 & 0 \\ \text { Eq.24 } & 0 & 0 & 0 & 0 & 0 & 0 & 0 & 0 & 0 & 0 & 0 & 0 & 0 & 0 & 0 & 0 & 0 & 0 & 0 & 0 & 0 & 0 & 1 & 0 & 1 & 0 & 1 & 0 & 0 & 0 & 0 & 0 \\ \text { Eq.25 } & 0 & 0 & 0 & 0 & 0 & 0 & 0 & 0 & 0 & 0 & 0 & 0 & 0 & 0 & 0 & 0 & 0 & 0 & 0 & 0 & 0 & 0 & 0 & 0 & 0 & 0 & 0 & 0 & 0 & 0 & 0 & 0 \\ \text { Eq.26 } & 0 & 0 & 0 & 0 & 0 & 0 & 0 & 0 & 0 & 0 & 0 & 0 & 0 & 0 & 0 & 0 & 0 & 0 & 0 & 0 & 0 & 0 & 0 & 0 & 0 & 0 & 0 & 0 & 0 & 0 & 0 & 0\end{array}$

Figura 4. Algoritmo de classificação das variáveis medidas

O algoritmo apresentado na Figura 4 possibilita verificar que algumas equações do modelo dependem diretamente das variáveis medidas chegando a solução somente pela observabilidade do sistema como, por exemplo, as Eqs. 12; 13; 16 a 21; 23 e 26. A Eq. 13 permite solucionar as Eqs. 16, 17, 18, 19, 20, 23 e 26 determinando-se as variáveis x14, x15, x16, x17, x29 e x32. E por conter variáveis medidas soluciona-se ainda a Eq.21.

Algumas variáveis, entretanto, não podem ser observadas por serem divisores do processo: x17, x19, x20, x21, x22, x27 e x28. Algumas equações do modelo também não foram utilizadas (Eqs. 15, 22 e 24) por não possuírem medidas relevantes ao processo por se tratar apenas de equipamentos para acionamento de turbo máquinas.

O que ficou claro na classificação é que ainda não havia medição existente em alguns pontos importantes, o que tornava o sistema não reconciliável e, portanto, suscetível a falhas. O mais adequado foi inserir um grupo de sensores onde há maior necessidade, ou seja, nas correntes c14(x23), c15(x25) e c16(x27) representadas na OPER 6 que são as maiores consumidoras de vapor. 


\section{Caso 2}

Nos estudos de caso extraídos de Oliveira Jr. et al. (2011) [28] e Prata et al. (2010) [25], foram selecionados apenas o balanço mássico na operação do reator. O sistema foi controlado para operar em estado estacionário onde seus dados referiam a um ciclo de 2 horas de produção e setpoint de 122 medidas/hora, tendo sido analisadas 4 variáveis de entrada e 1 de saída. Cabe explicar que havia confiabilidade nesses dados medidos em virtude das condições de controle do processo, e, portanto, somente erros aleatórios contidos nas medições considerando a inexistência de erros grosseiros [28].

$\mathrm{Na}$ Tabela 5 são apresentados os resultados obtidos na reconciliação dos dados reais operando em estado estacionário do processo de polimerização. As variáveis medidas de entrada vazão de propeno fresco (me), vazão do catalisador (mCAT), vazão do co-catalisador (mTEA), vazão do doador de elétrons (mPEEB) e a variável de saída vazão de polipropileno (mPOL).

Tabela 5. Dados reconciliados com as funções robustas

\begin{tabular}{lccccc}
\hline & me(kg/h) & mCAT(kg/h) & mTEA(kg/h) & mPEEB(kg/h) & mPOL(kg/h) \\
\hline Medido & 26744,39 & 2,3891 & 27,0 & 4,57 & 26169,47 \\
R_NT & 26740,84 & 2,3886 & 26,9 & 4,57 & 26165,73 \\
R_NC & 26750,25 & 2,3918 & 27,0 & 4,57 & 26175,37 \\
\hline
\end{tabular}

As vazões medidas de propeno fresco, CAT, TEA, PEEB e POL que foram reconciliadas com as funções robustas obtiveram desempenho similar e favorável na reconciliação com os dados reais, o que demonstra não haver medidas contaminadas com erros grosseiros nas variáveis apresentadas. As funções robustas New Target e Normal Contaminada apresentaram uma melhor precisão nos ajustes dos dados. Entretanto, analisando com relação ao ambiente computacional, a New Target otimizou a função objetivo com um número de iterações 5 vezes menor em relação a Normal Contaminada.

Em relação às variáveis estimadas (não medidas) através das funções robustas os valores resultantes estavam de acordo com o balanço mássico do modelo no conjunto de medidas existentes, sendo que a New Target mais uma vez apresentou um menor número de iterações em relação a função objetivo o que já era esperado devido a sua característica de estimador Mredescendente com ponto de corte para a estimação das variáveis.

\section{CONCLUSÃO}

A etapa inicial da reconciliação de dados, que é a classificação das variáveis, mostrou que a identificação de forma inequívoca de todas as variáveis do sistema possui importância absoluta na resolução de problemas industriais gerados por erros sistemáticos. Através da simplificação de um processo observou-se que a falta de sensores em divisores necessários estavam tornando o processo vulnerável e menos eficiente. Já na reconciliação utilizando funções robustas e dados reais ao aplicar a estatística robusta, inicialmente em um pequeno conjunto de dados, os resultados confirmaram a inexistência de erros grosseiros e como otimizar com um melhor ajuste nas medidas.

\section{AGRADECIMENTOS}

Os autores agradecem a Universidade Federal de Sergipe e a Coordenação de Aperfeiçoamento de Pessoal de Nível Superior pelo apoio científico e financeiro para o estudo. 


\section{LISTA DE SÍMBOLOS}

\begin{tabular}{|c|c|}
\hline SÍMBOLOS & DESCRIÇÃO [UNIDADE] \\
\hline CAT & Massa de catalisador no reator $[\mathrm{kg}]$ \\
\hline$K_{d}$ & Constante de desativação do sítio catalítico $\left[\mathrm{h}^{-1}\right]$ \\
\hline$K_{p}$ & Constante cinética de reação $\left[\mathrm{kg}^{2} /\left(\mathrm{kg}^{2} \cdot \mathrm{h}\right)\right]$ \\
\hline $\boldsymbol{m}_{\text {cat }}$ & Vazão de alimentação de catalisador $[\mathrm{kg} / \mathrm{h}]$ \\
\hline$m_{e}$ & Vazão de alimentação propeno fresco (contaminado por propano) $[\mathrm{kg} / \mathrm{h}]$ \\
\hline$m_{P E E B}$ & Vazão de alimentação de $P E E B[\mathrm{~kg} / \mathrm{h}]$ \\
\hline$m_{P o l}$ & Vazão de polipropileno produzido $[\mathrm{kg} / \mathrm{h}]$ \\
\hline$m_{\text {Purga }}$ & Vazão de purga $[\mathrm{kg} / \mathrm{h}]$ \\
\hline$m_{T E A}$ & Vazão de alimentação $T E A[\mathrm{~kg} / \mathrm{h}]$ \\
\hline$P a$ & Massa de propano no reator $[\mathrm{kg}]$ \\
\hline$P e$ & Massa de propeno no reator $[\mathrm{kg}]$ \\
\hline PEEB & Massa de para-etoxi etil benzoato $[\mathrm{kg}]$ \\
\hline POL & Massa de polímero no reator $[\mathrm{kg}]$ \\
\hline TEA & Massa de Trietil alumínio no reator $[\mathrm{kg}]$ \\
\hline$\alpha$ & Fator de recirculação de TEA [adimensional] \\
\hline $\boldsymbol{w}_{a}$ & Fração mássica de propano na corrente de propeno $[\mathrm{kg} / \mathrm{kg}]$ \\
\hline$\rho_{a}$ & Densidade do propano $[\mathrm{kg} / \mathrm{L}]$ \\
\hline$\rho_{e}$ & Densidade do propeno $[\mathrm{kg} / \mathrm{L}]$ \\
\hline$\rho_{p o l}$ & Densidade do polímero $[\mathrm{kg} / \mathrm{L}]$ \\
\hline
\end{tabular}

\section{REFERÊNCIAS BIBLIOGRÁFICAS}

1. Wu S, Ye Q, Gu X. Research on data reconciliation based on generalized T distribution with historical data. Neuroc. 2016 Jan;175(part A): 808-815, doi: 10.1016/j.neurocom.10.093.

2. Kuehn DR, Davidson H. Computer control. II. Mathematics of control. Chem. Eng. Prog. 1961 Jan; 57(6):44.

3. Mah RSH, G. Stanley D. Downing. Reconciliation and rectification of process flow and inventory data. Ind. Chem. Proc. Des. Dev. 1976 Jan;15:175-183, doi: 10, 1021/I260057A030.

4. Hodouin D, Everell MD. A hierarchical procedure for adjustment and material balancing of mineral processes data. Int. J. Miner. Process. 1980 Set;7:91-116, doi: 10.1016/0301-7516(80)90002-2.

5. Crowe CM, Campos YAG, Hrymak A. Reconciliation of process flow rates by matrix projection. Part I: Linear case. AIChE Journ. 1983 Nov;29:881-888, doi: 10.1002/AIC.690290602.

6. Crowe CM. Reconciliation of process flow rates by matrix projection. Part II: Nonlinear case. AIChE Journ. 1986 Abr;32:616-623, doi: 10.1002/AIC.690320410.

7. Ripps DL. Adjustment of experimental data. Chem. Eng. Progress - Symp. Series. 1965 Set;61:8-13.

8. Vaclavek V. Studies on system engineering - III optimal choice of the balance measurements in complicated chemical engineering systems. Chem. Eng. Sci. 1969 Jun;24(6):947-955, doi: 10.1016/00092509(69)87003-X.

9. Vaclavek V. Testing of over-and underdetermination of problem formulations for simulation calculations of a complex chemical process. Chem. Eng. Sci. 1972 Set;27(9):1669-1676, doi: 10.1016/0009-2509(72)80081-2.

10. Romagnoli JA, Stephanopoulos G. Rectification of process measurements data in the presence of gross errors. Chem. Eng. Sci. 1981 Abr;36(11):1849-63, doi: 10.1016/0009-2509(81)80133-9.

11. Václavek V, Loucka M. Selection of measurements necessary to achieve multicomponent mass balances in chemical plants. Chem. Eng. Sci. 1976 Mar;31:1199-205, doi: 10.1016/0009-2509(76)850294

12. Bagajewicz MJ. Process plant instrumentation: Design and upgrade. Technomic Publishing Company, Pennsylvania, 2001 Nov; 205p.

13. Stanley G, Mah RS. H. Observability and redundancy in process data estimation. Chem. Eng. Sci. 1981a Jun;36:259-83, doi: 10.1016/0009-2509(81)85004-X.

14. Stanley G, Mah RSH. Observability and redundancy classification in process networks. Chem. Eng. Sci. 1981b Abr;36:1941-54, doi: 10.1016/0009-2509(81)80034-6.

15. Oliveira Jr AM, Pinto JCCS, Lima EL. A identificação estrutural como proposta de classificação de variáveis para a reconciliação de dados industriais. Scientia Plena. 2011 Nov;7(11). 
16. Arora N, Biegler LT. Redescending estimators for data reconciliation and parameter estimation. Computers and Chemical Engineering. 2001 Out;25:1585-1599, doi: 10.1016/S0098-1354(01)00721-9.

17. Ozyurt DB, Pike RW. Theory and practice of simultaneous data reconciliation and gross error detection for chemical process. Comp. and Chem. Eng. 2004 Mar;28:381-402, doi: 10.1016/j.compchemeng.2003.07.001.

18. Zhang Z, Shao Z, Chen X, Wang K, Qian J. Quasi-weighted least squares estimator for data reconciliation. Comp. and Chem. Eng. $2010 \mathrm{Fev} ; 34: 154-162$, doi: 10.1016/j.compchemeng.2009.09.007. 19. Bourouis M, PI-bouleau L, Floquet P. Simulation and data validation in multistage flash desalination plants. Des. 1998 Mar;115:1-14. doi: 10.1016/S0011-9164(98)00021-6.

20. Schladt M, Hu B. Soft sensor based on nonlinear steady-state data reconciliation in the process industry. Chem. Eng. and Proc. 2007 Nov;46:1107-1115, doi: 10.1016/j.cep.2006.06.022.

21. Lid T, Skogestad S. Data reconciliation and optimal of a catalytic Naphtha Reformer. Journ. of Proc. Cont. 1998 Mar;18:320-331, doi: 10.1016/j.jprocont.2007.09.002.

22. Chen J, Peng Y, Munoz JC. Correntropy estimator for data reconciliation. Chem. Eng. Sci. 2013 Dez; 104:1019-1027, doi: 10.1016/j.ces.2013.09.034.

23. Jin S, Hung LX, Liu M. A New Target function for robust data reconciliation. Ind. and Eng. Chem. Res. 2012 Jul;51:10220-10224, doi: 10.1021/ie2030773.

24. Prata DM, Pinto JC, Lima EL. Comparative analysis of robust estimators on nonlinear dynamic data reconciliation. Comp. Aid. Chem. Eng. 2008 Out;25:501-506, doi: 10.1016/S1570-7946(08)80088-0.

25. Prata DM, Schwaab M, Lima EL, Pinto JC. Simultaneous robust data reconciliation and gross error detection through particle swarm optimization for an industrial polypropylene reactor. Chem. Eng. Sci. 2010 Set;65(17):4943-4954, doi: 10.1016/j.ces.2010.05.017.

26. Huber PJ. Robust statistics. New York, John Wiley, 1981 Mar.

27. Hampel FR. The breakdown points of the mean combined with some rejection rules. Tech. 1985 Mar;27:95-107, doi: 10.2307/1268758

28. Oliveira Jr AM, Pinto JCCS, Lima EL. Uma proposta para detecção de erros grosseiros em balanços de massa de processos industriais. Scientia Plena, 2011 Abr;7(4). 